\title{
Default mode network connectivity in patients with idiopathic normal pressure hydrocephalus
}

\author{
Hui Ming Khoo, MD, PhD, ${ }^{1}$ Haruhiko Kishima, MD, PhD, ${ }^{1}$ Naoki Tani, MD, PhD, ${ }^{1}$ \\ Satoru Oshino, MD, PhD, ${ }^{1}$ Tomoyuki Maruo, MD, PhD, ${ }^{1}$ Koichi Hosomi, MD, PhD, ${ }^{1,2}$ \\ Takufumi Yanagisawa, MD, PhD, ${ }^{1}$ Hiroaki Kazui, MD, PhD, ${ }^{3}$ Yoshiyuki Watanabe, MD, PhD, ${ }^{4}$ \\ Toshio Shimokawa, PhD, ${ }^{5}$ Toshihiko Aso, MD, PhD, ${ }^{6}$ Atsushi Kawaguchi, PhD, ${ }^{7}$ \\ Fumio Yamashita, PhD, ${ }^{8}$ Youichi Saitoh, MD, PhD, ${ }^{1,2}$ and Toshiki Yoshimine, MD, PhD ${ }^{1}$
}

Departments of ${ }^{1}$ Neurosurgery; ${ }^{2}$ Neuromodulation and Neurosurgery, Center of Medical Innovation and Translational Research; ${ }^{3}$ Psychiatry; and ${ }^{4}$ Radiology; Osaka University Graduate School of Medicine, Suita, Osaka Prefecture; ${ }^{5}$ Department of Ecosocial System Engineering, Graduate School of Medicine and Engineering, University of Yamanashi, Kofu City, Yamanashi Prefecture; ${ }^{6}$ Human Brain Research Center, Graduate School of Medicine, Kyoto University; ${ }^{7 B i o m e d i c a l ~ S t a t i s t i c s ~ a n d ~ B i o i n f o r m a t i c s, ~}$ Graduate School of Medicine and Faculty of Medicine, Kyoto University, Kyoto, Kyoto Prefecture; and ${ }^{8}$ Division of Ultrahigh Field MRI, Institute for Biomedical Sciences, Iwate Medical University, Iwate Prefecture, Japan

OBJECTIVE Idiopathic normal pressure hydrocephalus (iNPH) is a neurological disorder characterized by gait disturbance, cognitive impairment, and incontinence. It is unclear whether the pathophysiology of iNPH is associated with alterations in the default mode network (DMN). The authors investigated alterations in the DMN of patients with iNPH and sought to determine whether a relationship exists between the resting-state functional connectivity of the DMN and a patient's clinical symptoms.

METHODS Resting-state functional MRI (rs-fMRI) was performed in 16 preoperative patients with iNPH and 15 neurologically healthy control subjects of a similar age. Independent component and dual-regression analyses were used to quantify DMN connectivity. The patients' clinical symptoms were rated according to the iNPH grading scale (iNPHGS). Each of their specific clinical symptoms were rated according to the cognitive, gait, and urinary continence domains of iNPHGS, and neurocognitive status was assessed using the Mini-Mental State Examination, Frontal Assessment Battery (FAB), and Trail Making Test Part A. The strength of DMN connectivity was compared between patients and controls, and the correlation between DMN connectivity and iNPHGS was examined using both region of interest (ROI)-based analysis and voxel-based analysis. The correlation between DMN connectivity and each of the specific clinical symptoms, as well as neurocognitive status, was examined using voxel-based analysis.

RESULTS Both ROI-based and voxel-based analyses revealed reduced DMN connectivity in patients with iNPH. ROI-based analysis showed increased DMN connectivity with worsening clinical symptoms of iNPH. Consistently, voxelbased analyses revealed that DMN connectivity correlated positively with the iNPHGS score, as well as the cognitive and urinary continence domain scores, and negatively with the FAB score. The significant peak in correlation in each case was localized to the precuneus.

CONCLUSIONS This is the first study to establish alterations in the DMN of patients with iNPH. DMN connectivity may be a useful indicator of the severity of clinical symptoms in patients with iNPH.

http://thejns.org/doi/abs/10.3171/2015.1.JNS141633

KEY WORDS default mode network; idiopathic normal pressure hydrocephalus; resting-state functional MRI; functional connectivity

ABBREVIATIONS AD = Alzheimer's disease; DMN = default mode network; EPI = echo-planar imaging; FAB = Frontal Assessment Battery; FWE = family-wise error; ICA $=$ independent component analysis; iNPH = idiopathic normal pressure hydrocephalus; iNPHGS $=$ iNPH grading scale $(c-i N P H G S=$ cognitive domain, $g$-iNPHGS $=$ gait domain, and i-iNPHGS = urinary continence domain); MELODIC = Multivariate Exploratory Linear Optimized Decomposition into Independent Components; MMSE = MiniMental State Examination; ROI = region of interest; rs-fMRI = resting-state functional MRI; SPM8 = Statistical Parametric Mapping-8; TMT-A = Trail Making Test Part A. SUBMITTED July 14, 2014. ACCEPTED January 29, 2015.

INCLUDE WHEN CITING Published online August 21, 2015; DOI: 10.3171/2015.1.JNS141633. 
I DIOPATHIC normal pressure hydrocephalus (iNPH) is a neurological disorder that preferentially affects the elderly. ${ }^{22} \mathrm{iNPH}$ is characterized by the clinical triad of gait disturbance, cognitive impairment, and urinary incontinence. Diagnosis is based on the patient's clinical symptoms and is supported by specific neuroradiological features, e.g., enlarged ventricles, tightness of the high parietal convexity, and disproportionately enlarged subarachnoid spaces. ${ }^{18}$ Results of the CSF tap test can also support the diagnosis. ${ }^{18}$ Although iNPH is well known, the neural networks and underlying pathophysiological mechanisms of this neurological disorder remain unclear.

In recent years, spontaneous brain activity and its relation to cognition and behavior have been studied extensively. ${ }^{20}$ Resting-state functional MRI (rs-fMRI) studies have shown alterations in connectivity within the default mode network (DMN) to be associated with cognitive impairments in several disorders, e.g., Alzheimer's disease (AD), multiple sclerosis, traumatic brain injury, and attention deficit hyperactivity disorder. 4,5,10,12,27,28 However, no studies have investigated alteration of DMN connectivity in iNPH patients.

The aim of this study was to investigate the pathophysiological changes within the neural network underlying iNPH. We hypothesized that 1) the pathophysiology of this disease is associated with abnormalities in DMN connectivity and 2) the progression of clinical symptoms is related to changes in DMN connectivity. We tested these hypotheses by comparing DMN connectivity between patients with iNPH and neurologically healthy persons of a similar age (control subjects) and investigating whether DMN connectivity correlates with the severity of clinical symptoms. We explored these questions using independent component analysis (ICA) of the rs-fMRI data obtained from the patients and control subjects.

\section{Methods \\ Study Groups}

Seventeen consecutive patients with iNPH and 15 neurologically healthy persons of a similar age were recruited for the study. Inclusion criteria for the patients were 1) age $>60$ years; 2) presentation of at least 1 of the 3 symptoms of the classic triad (gait disturbance, cognitive dysfunction, or urinary incontinence); 3) ventricular enlargement with a narrowing of the high convexity and midline subarachnoid spaces on MR images; 4) absence of any other disorder that can cause ventricular dilation or the presenting symptoms; 5) CFS pressure $<20 \mathrm{~cm} \mathrm{H}_{2} \mathrm{O}$ and normal CSF content; and 6) transient improvement of symptoms after the CSF tap test. Improvement of symptoms was confirmed in all patients after lumboperitoneal shunting, which was performed after acquiring rs-fMRI data. This means all study patients fulfilled the criterion for definite iNPH in accordance with the Japanese guidelines for the management of iNPH. ${ }^{14,18}$ Patients suspected of having another neurological disorder, such as Parkinson's disease, $\mathrm{AD}$, or cerebrovascular disease, were excluded on the basis of neurological and neuropsychological examinations by trained psychiatrists and neurologists at our hospital. All control subjects were screened to ensure that they were free of any sign or symptom of iNPH or other neurological disorder, as described above.

One of the 17 patients was excluded because of MRI motion artifacts. Thus, the final patient group consisted of 16 patients ( 7 men and 9 women; age range 67-84 years, mean age $75 \pm 5.5$ years). The control group comprised 15 healthy persons ( 9 men and 6 women; age range 61-83 years, mean age $71.2 \pm 6.9$ years). The clinical characteristics of the patients are summarized in Table 1.

\section{Standard Protocol Approval and Consent}

The ethics committee of Osaka University Hospital approved the study protocol. Each patient, or a family member, and each control subject provided written informed consent for participation in the study.

\section{Neuropsychological Assessment}

Each patient's clinical symptoms were rated according to the iNPH grading scale (iNPHGS). ${ }^{16,18}$ On the iNPHGS, each of the clinical triad symptoms is scored from 0 to 4 , with the total score ranging from 0 (normal) to 12 (severe). The overall severity of each symptom was rated using the cognitive, gait, and urinary continence domains of the iNPHGS (c-iNPHGS, g-iNPHGS, and i-iNPHGS, respectively). Each patient's neurocognitive status was evaluated by trained neuropsychologists using the following neuropsychological assessment instruments: 1) Mini-Mental State Examination (MMSE) for global cognitive function (score range 0-30) (higher scores indicate better functioning); 2) Frontal Assessment Battery ${ }^{13}$ (FAB) for attention and executive function (score range 0-18) (higher scores indicate better functioning); and 3) Trail Making Test Part $\mathrm{A}^{23}$ (TMT-A) for psychomotor speed involving focused attention, visual scanning, and motor planning (completion time in seconds) (higher scores indicate poorer functioning).

\section{MRI Data Acquisition}

MRI was performed using a GE Signa 3-Tesla Excite HDxt scanner at Osaka University Hospital. For functional images, axial slices were obtained in a single-shot gradient echo-planar imaging (EPI) sequence for each subject (TR $2000 \mathrm{msec}$, TE $30 \mathrm{msec}$, flip angle $90^{\circ}$, FOV $220 \mathrm{~mm}$, voxel size $3.4 \times 3.4 \times 3.5 \mathrm{~mm}$ ). A 5-minute continuous resting-state scan was acquired for each subject. Subjects were instructed to relax, stay awake, lie still, and think of nothing in particular while keeping their eyes open. One hundred fifty whole-brain EPI volumes were acquired. The first 5 images in each run were discarded to allow magnetization to reach equilibrium.

A high-resolution T1-weighted structural image scanned with an inversion recovery 3D spoiled GRASS (Gradient Recall Acquisition using Steady States) sequence (TR $7.0 \mathrm{msec}$, TE $2.9 \mathrm{msec}$, TI $400 \mathrm{msec}$, FOV $240 \mathrm{~mm}$; 1-mm isotropic voxel) was acquired in the same session.

\section{Image Preprocessing and Analysis}

The analytical pipeline is illustrated in Fig. 1. Imaging data were processed by MATLAB (version 8.0, MathWorks, Inc.). Analysis was performed with Statistical 
TABLE 1. Clinical characteristics, neuropsychological assessment scores, and DMN connectivities of the study patients $(n=16)^{*}$

\begin{tabular}{|c|c|c|c|c|c|c|c|c|c|c|}
\hline Case No. & Age (yrs) & Sex & iNPHGS & c-iNPHGS & g-iPNHGS & i-iNPHGS & MMSE & FAB & TMT-A & $\begin{array}{c}\text { DMN Connectivity } \\
\text { (z score) }\end{array}$ \\
\hline 1 & 71 & $\mathrm{~F}$ & 4 & 2 & 2 & 0 & 26 & 16 & 71 & 2.12 \\
\hline 2 & 67 & $\mathrm{~F}$ & 6 & 2 & 2 & 2 & 24 & 12 & 80 & 2.52 \\
\hline 3 & 80 & $\mathrm{~F}$ & 8 & 3 & 2 & 3 & 19 & 10 & 151 & 3.05 \\
\hline 4 & 77 & $\mathrm{M}$ & 8 & 3 & 2 & 3 & 23 & 5 & 395 & 2.54 \\
\hline 5 & 80 & $F$ & 7 & 3 & 2 & 2 & 17 & 6 & 183 & 2.36 \\
\hline 6 & 67 & $\mathrm{~F}$ & 9 & 3 & 3 & 3 & 20 & 4 & 350 & 2.40 \\
\hline 7 & 75 & $\mathrm{M}$ & 5 & 2 & 2 & 1 & 23 & 14 & 114 & 1.83 \\
\hline 8 & 68 & $\mathrm{M}$ & 6 & 2 & 2 & 2 & 27 & 12 & 60 & 1.34 \\
\hline 9 & 75 & $M$ & 3 & 1 & 2 & 0 & 27 & 16 & 50 & 1.66 \\
\hline 10 & 74 & $\mathrm{~F}$ & 8 & 3 & 3 & 2 & 17 & 6 & 231 & 2.19 \\
\hline 11 & 81 & $\mathrm{M}$ & 9 & 3 & 3 & 3 & 14 & 8 & 180 & 2.87 \\
\hline 12 & 69 & $\mathrm{~F}$ & 3 & 2 & 1 & 0 & 22 & 11 & 142 & 1.62 \\
\hline 13 & 77 & $\mathrm{~F}$ & 8 & 2 & 3 & 3 & 26 & 9 & 110 & 2.82 \\
\hline 14 & 81 & $\mathrm{~F}$ & 9 & 3 & 3 & 3 & 10 & - & - & 2.24 \\
\hline 15 & 78 & $\mathrm{M}$ & 6 & 2 & 2 & 2 & 25 & 11 & 167 & 2.00 \\
\hline 16 & 82 & $\mathrm{M}$ & 4 & 1 & 1 & 2 & 28 & 17 & 40 & 1.54 \\
\hline Mean \pm SD & $75.1 \pm 5.3$ & & & & & & $21.8 \pm 5.2$ & $10.5 \pm 4.2$ & $155 \pm 104$ & $2.19 \pm 0.50$ \\
\hline Median (range) & & & $6.5(2-9)$ & $2.0(1-3)$ & $2.0(1-3)$ & $2.0(0-3)$ & & & & \\
\hline
\end{tabular}

- = data not available.

* Because 1 patient did not complete the FAB and TMT-A assessments, all analyses involving these assessments were based on the data from 15 patients.
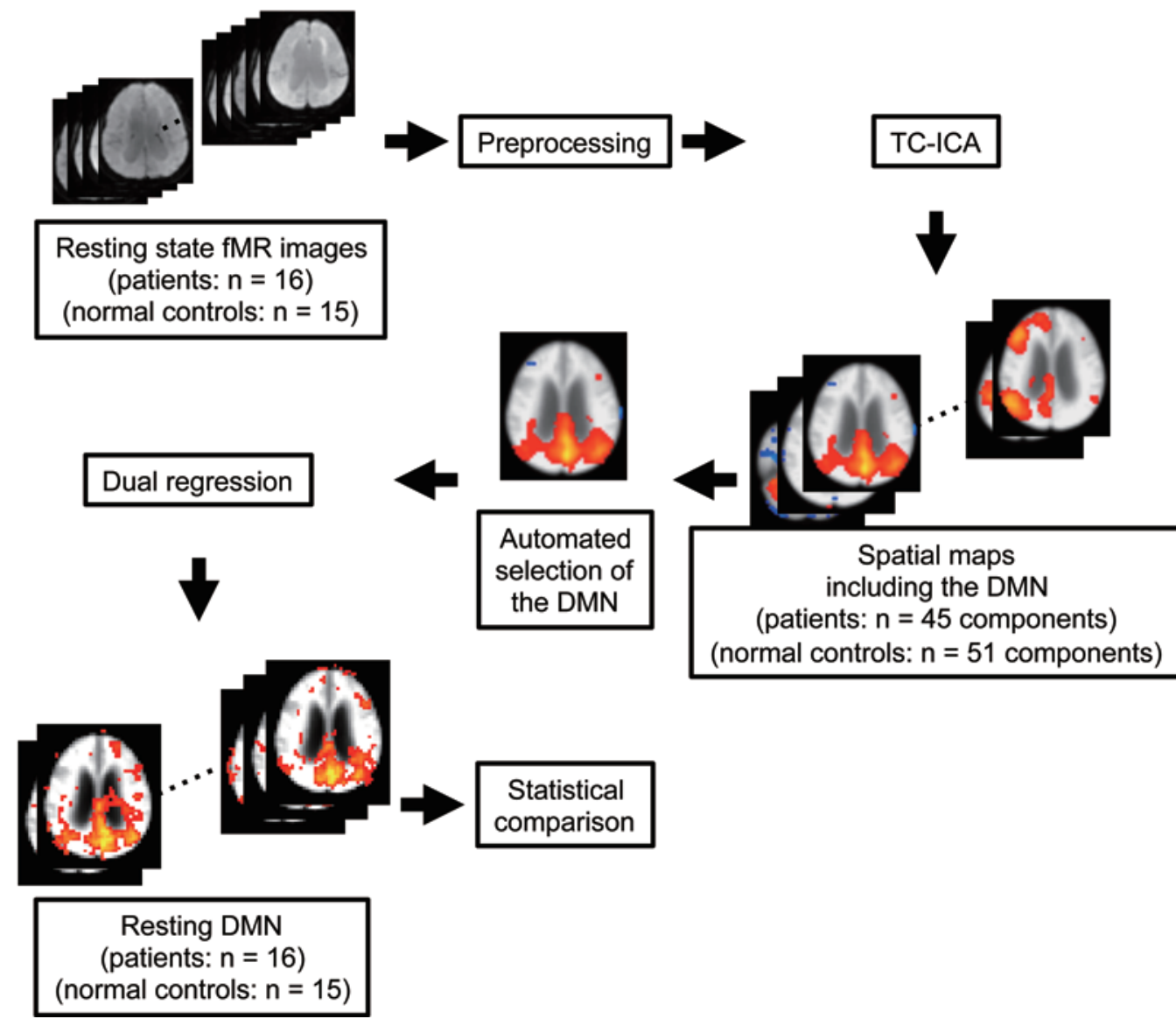

FIG. 1. Overview of the analytical pipeline of this study used to produce subject-specific DMN connectivity maps for all subjects. $\mathrm{fMR}=$ functional MR; TC-ICA = temporal concatenation group-independent component analysis. 

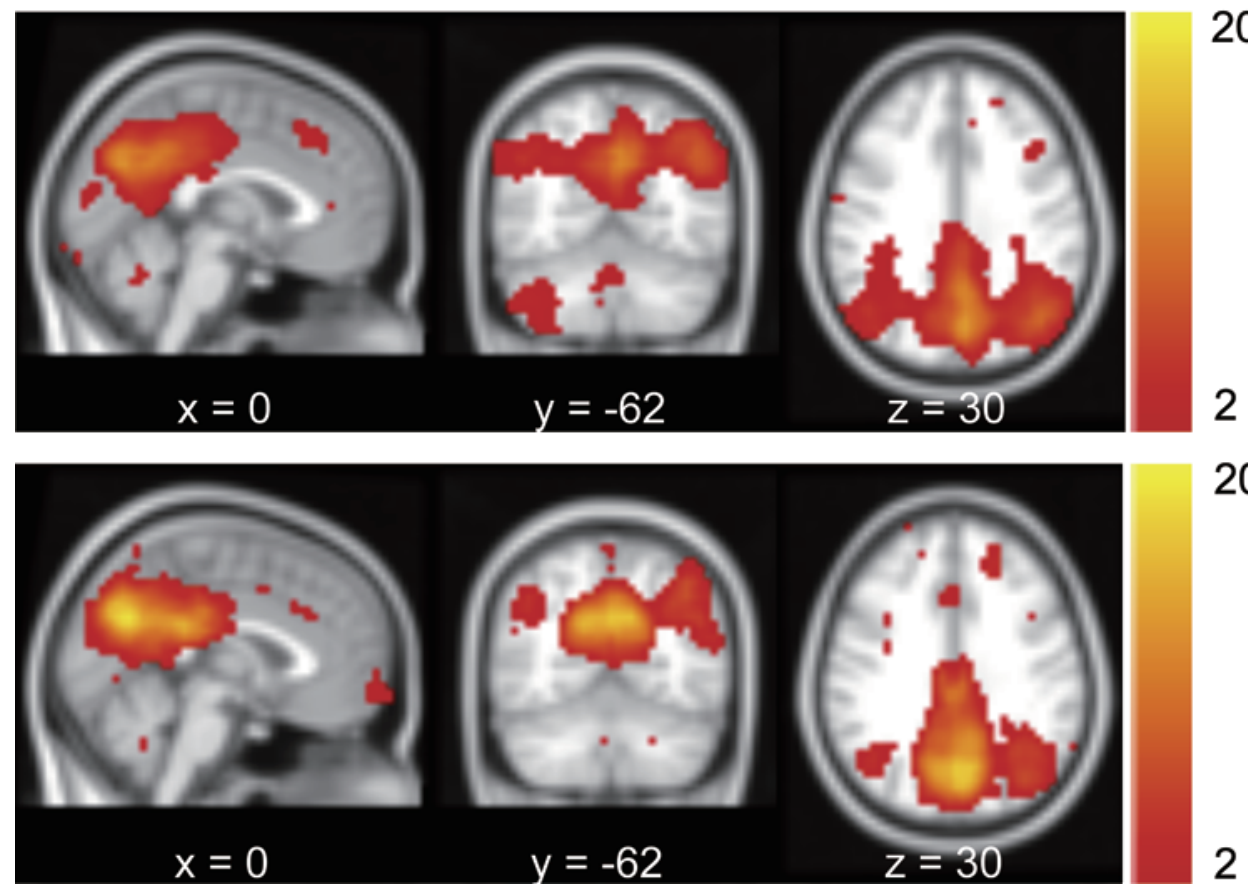

FIG. 2. DMN identified on TC-ICA. Upper: Patients with iNPH. Lower: Control subjects of similar age. Results are superimposed on an MNI152 3.5-mm template in the radiological convention (right-left).

Parametric Mapping-8 (SPM8) (www.fil.ion.ucl.ac.uk/ spm) and probabilistic ICA ${ }^{2}$ as implemented in the Multivariate Exploratory Linear Optimized Decomposition into Independent Components (MELODIC) ${ }^{1}$ part of the FMRIB Software Library (www.fmrib.ox.ac.uk/fsl).

Structural images were first skull stripped using SPM8. Functional image analysis was carried out using MELODIC. Individual prestatistical processing consisted of motion correction, removal of nonbrain structures, spatial smoothing with a gaussian kernel of full width at half maximum of $5 \mathrm{~mm}$, and high-pass temporal filtering equivalent to 100 seconds $(0.01 \mathrm{~Hz})$. The functional images were registered to the individual's skull-stripped structural images and the older adult-based MNI152 standard space image (http://www.mccauslandcenter.sc.edu/ CRNL/clinical-toolbox) ${ }^{21}$ using FMRIB's Nonlinear Image Registration Tool. A single 4D data set was created for each study group by temporal concatenation of the preprocessed functional images representing 145 time points for each subject in each respective study group.

The 4D data sets were decomposed using ICA to identify large-scale patterns of functional connectivity in each study group. We allowed MELODIC to use a probabilistic principal component model to estimate the optimal number of components needed to decompose the data., ${ }^{1,3}$ The data sets were decomposed into 45 components in the patient group and 51 components in the control group, with each independent component representing either a resting state network or an artifact. ${ }^{7}$ For unbiased identification of the DMN, the component that most closely matched the DMN was selected for each group using an automated 2-step process called the "goodness-of-fit" approach. . $^{10,24}$ The standard DMN template we used to select the "best fit" was generated from the 20-dimensional ICA BrainMap components downloaded from the FMRIB website (http://fsl.fmrib.ox.ac.uk/analysis/brainmap+rsns/). ${ }^{26}$ The selected DMN of each group (Fig. 2) was then subjected to subsequent analyses.

The dual-regression approach was used to identify subject-specific time courses and spatial maps of the DMN ${ }^{8}$ The intensity of a given voxel in the spatial map reflects the degree to which the time series of the voxel correlates with the time series of the DMN. Thus, the component intensities of an image constitute the subject-specific connectivity map of the DMN. This technique normalizes intensity across images to allow direct statistical comparisons between images. We are thereby able to compare DMN connectivity between subjects and test for a relationship between DMN connectivity and individual cognitive function.

\section{Between-Group Comparisons and Correlation Analyses Region of Interest-Based Analysis}

To create a DMN region of interest (ROI) for each group, 1-sample t-tests were performed on the subjectspecific spatial DMN maps for each group. The resultant $\mathrm{t}$ values were thresholded at an uncorrected $\mathrm{p}<0.001$, and the DMN ROIs were then identified as clusters that reached a significance threshold of $p<0.05$ after correction for multiple comparisons by means of the wholebrain topological family-wise error (FWE) probability implemented in SPM8. We then summarized the subjectspecific DMN connectivity map by averaging the voxels within the respective group DMN ROI. The value obtained was used as a measure of DMN connectivity for each subject. ${ }^{15}$ We used the 2-sample t-test to compare 
DMN connectivity between patients and control subjects. We used Pearson's correlation coefficient to identify any relationship between the patient's DMN connectivity and iNPHGS scores. Because DMN connectivity is reported to correlate with age, ${ }^{6}$ 2-tailed correlation coefficients were also calculated to control for patient age. Statistical analyses were performed using SPSS statistical software (version 21.0, SPSS for Mac), and $\mathrm{p}<0.05$ was considered statistically significant.

\section{Voxel-Based Analysis}

We entered the subject-specific DMN connectivity map into the group analysis using a random-effects model (SPM8). All voxel-based analyses were performed within the area of the DMN ROI for each group. Voxel-wise comparisons of the patient- and control subject-specific DMN connectivity maps were performed using a general linear model and 2-sample t-test. We tested for differences between groups for each contrast of interest. Voxelwise regression analysis of each patient's subject-specific DMN connectivity map was performed using general linear modeling, with each of the iNPHGS, c-iNPHGS, g-iNPHGS, i-iNPHGS, MMSE, FAB, and TMT-A scores entered as explanatory variables. To control for patient age, age was introduced into the statistical model as a covariate of no interest. Statistical parametric maps were first thresholded at an uncorrected $t \leq 2.3$. We then identified the clusters that achieved a significance threshold of whole-brain topological FWE-corrected $\mathrm{p}<0.05$ (Table 2 ). We used this uncorrected threshold because there were 0 voxels that passed the corrected threshold for each of the scores. Lastly, the mean z score of the voxels within the significant clusters was correlated against the score for each of the explanatory variables. Pearson's and Spearman's correlation coefficients were calculated for the continuous and categorical variables, respectively.

\section{Results \\ Reduced Resting-State Connectivity of DMN in iNPH Patients}

ROI-based analysis revealed a significant difference in DMN connectivity between patients with iNPH and neurologically healthy control subjects of a similar age (Fig. 3 upper). Reduced DMN connectivity was observed in patients in comparison with that in control subjects $(\mathrm{p}<$ 0.001). Voxel-based analysis revealed reduced functional connectivity between the posterior cingulate cortex/precuneus and the rest of the DMN nodes in iNPH patients in comparison with the control subjects (Fig. 3 lower).

\section{Correlation Between DMN Connectivity and Severity of iNPH Symptoms}

ROI-based analysis revealed a significant positive correlation between DMN connectivity and iNPHGS scores $(\mathrm{r}=0.72 ; \mathrm{p}=0.002)$ (Fig. 4), that is, DMN connectivity increased with worsening clinical symptoms of iNPH. This correlation remained significant after correcting for age $(\mathrm{r}$ $=0.70 ; \mathrm{p}=0.004)$.

Because a significant correlation was found between DMN connectivity and the iNPHGS scores in the ROI-
TABLE 2. Results of the voxel-based analysis: voxels within clusters showing a correlation between DMN connectivity and assessment scores*

\begin{tabular}{lcrrrrr}
\hline \multirow{2}{*}{$\begin{array}{l}\text { Assessment } \\
\text { Instrument }\end{array}$} & $\begin{array}{c}\text { Anatomical } \\
\text { Region }\end{array}$ & \multicolumn{1}{c}{ Coordinates (mm) } & Cluster & $\begin{array}{c}\mathrm{p} \text { Value } \\
\text { (FWE) }\end{array}$ \\
\cline { 3 - 6 } iNPHGS & Precuneus & -15 & -58 & 34 & 249 & $<0.001$ \\
\hline & & 6 & -66 & 16 & & \\
\hline c-iNPHGS & Precuneus & -1 & -72 & 24 & 81 & 0.033 \\
\hline & & -8 & -62 & 20 & & \\
\hline i-iNPHGS & Precuneus & -18 & -72 & 27 & 163 & 0.001 \\
\hline & & -15 & -72 & 16 & & \\
\hline & & -4 & -34 & 41 & & \\
\hline FAB & Precuneus & -12 & -80 & 20 & 121 & 0.006 \\
\hline & & 10 & -76 & 24 & & \\
\hline & & -4 & -76 & 24 & & \\
\hline
\end{tabular}

$\mathrm{MNI}=$ Montreal Neurological Institute.

* Only assessment scores with clusters passing the significance threshold (topological FWE of 0.05 ) are shown. Within each cluster, the voxels with the top $3 \mathrm{t}$ values and their approximate anatomical location are listed (according to the Harvard-Oxford atlas).

based analysis, we conducted voxel-based analyses to explore the correlation between DMN connectivity, iNPHGS score, each of the iNPH-specific clinical symptoms (ciNPHGS, g-iNPHGS, and i-iNPHGS), and the scores of each neuropsychological assessment (MMSE, FAB, and TMT-A). DMN connectivity consistently showed positive voxel-based correlations with the iNPHGS score (cluster size $=249 ; \mathrm{p}<0.001$ ), $\mathrm{c}-\mathrm{iNPHGS}$ score $($ cluster size $=81$; $\mathrm{p}=0.033$ ), and $\mathrm{i}$-iNPHGS score (cluster size $=163 ; \mathrm{p}=$ 0.001 ), and a negative voxel-based correlation with the FAB score (cluster size $=121 ; p=0.006$ ). The significant peak in correlation was localized to the precuneus in each case (Fig. 5 and Table 2). DMN connectivity did not correlate significantly with the g-iNPHGS, MMSE, or TMT-A score.

\section{Discussion}

To our knowledge, this is the first study to examine intrinsic brain activity in the DMN of patients with iNPH using rs-fMRI. rs-fMRI offers the advantage of not requiring the use of any task to probe the function of a brain network. In patients with iNPH - who are cognitively impaired and may not be able to appropriately perform specific tasksrs-fMRI provides a good way for clinicians to understand the pathophysiology of this neurological disorder. ${ }^{17}$

We demonstrated a reduction in DMN connectivity in patients with iNPH compared with neurologically healthy control subjects. This is consistent with the abnormalities observed in other geriatric dementias, including AD and mild cognitive impairment. ${ }^{5}$ Reduced connectivity in the DMN is reportedly associated with cognitive decline in a number of neurological disorders. . $^{5,30}$

Furthermore, our results revealed that DMN connectiv- 

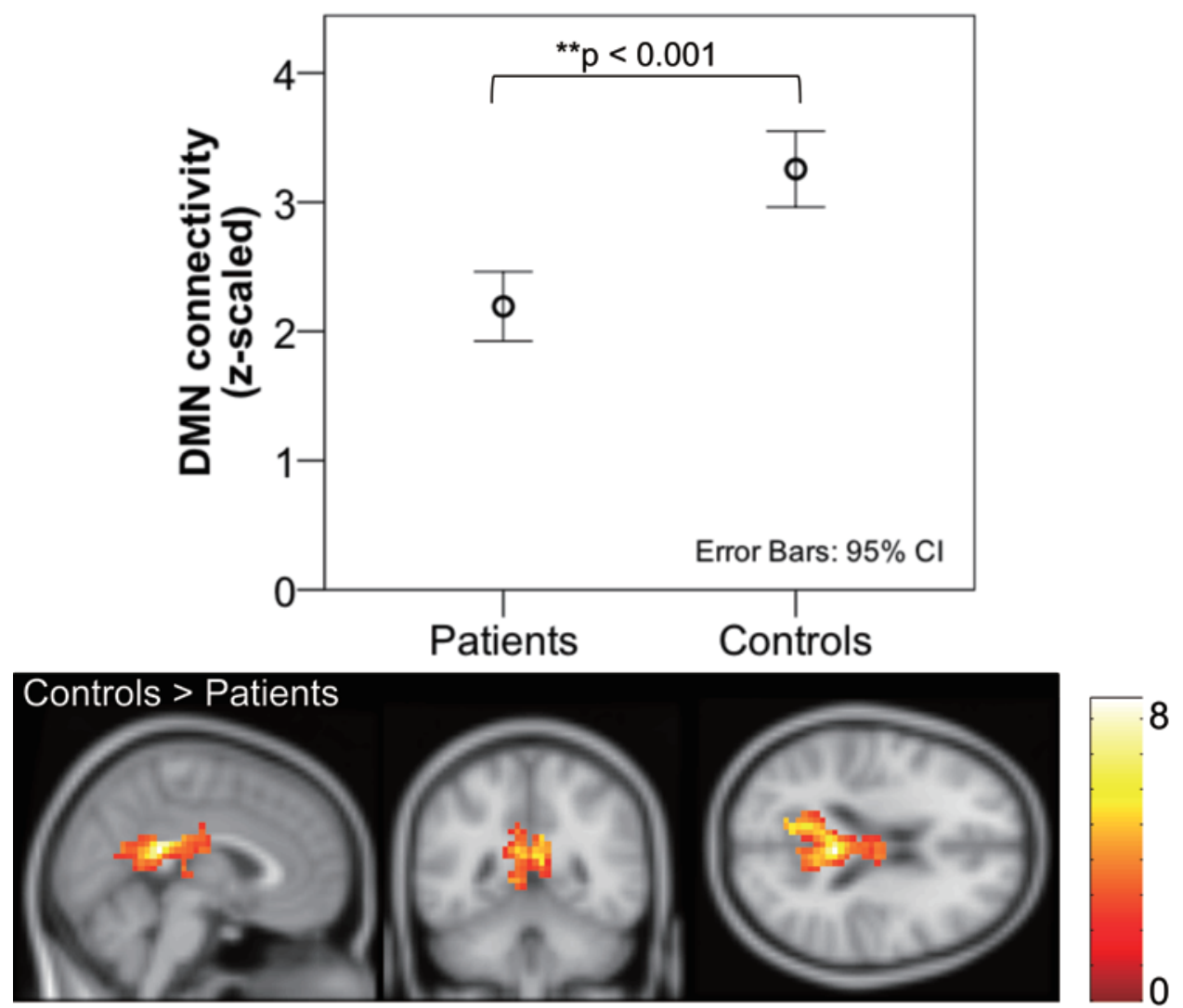

FIG. 3. Upper: Average and 95\% confidence interval of connectivity within the DMN ROls for patients with iNPH and neurologically healthy control subjects of a similar age. Lower: Voxels, which show greater DMN connectivity in neurologically healthy control subjects of a similar age in comparison to patients with iNPH, are shown in red-yellow and superimposed on an MNI152 3.5-mm template in the radiological convention (right-left).

ity correlates with the severity of iNPH symptoms. The reduced DMN connectivity may thus reflect a compensatory neural process. ${ }^{25} \mathrm{DMN}$ connectivity might decrease to compensate for impaired cognition, attention, gait, and

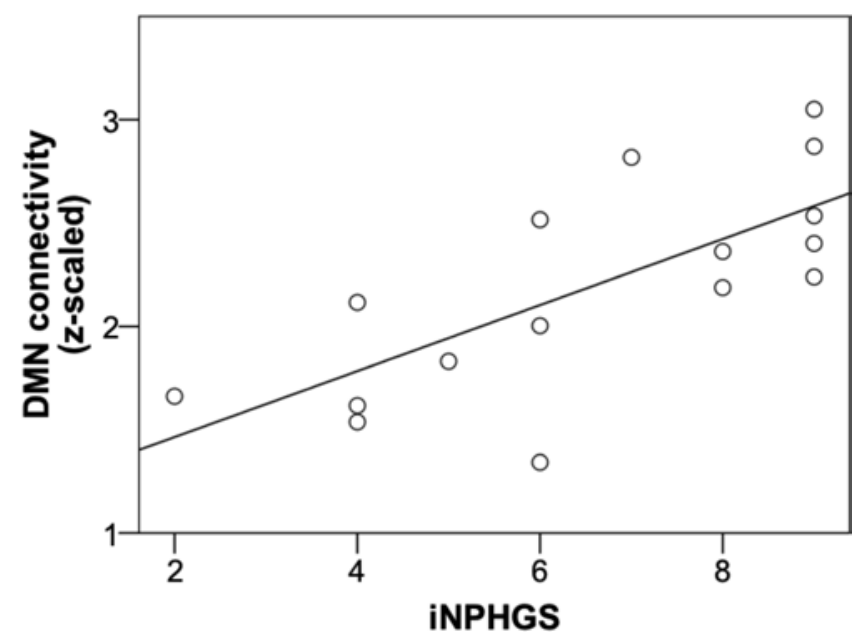

FIG. 4. Correlation between average connectivity within DMN ROls and the severity of clinical symptoms as measured by the iNPHGS in patients with iNPH. DMN connectivity increases with the increasing severity of clinical symptoms, which is indicated by the increasing iNPHGS scores. continence in patients in the mild iNPH stage. With the patient's worsening symptoms and decline to the severe iNPH stage, the compensatory decrease in DMN connectivity may not be maintained, resulting in an increase in DMN connectivity relative to that in the mild iNPH stage. However, further studies that combine rs-fMRI and taskbased fMRI may be necessary to confirm this postulation.

A significant peak in correlation between DMN connectivity and severity of clinical symptoms was localized to the precuneus, suggesting the importance of the precuneus within the DMN architecture in patients with iNPH. In line with previous reports, these findings indicate that the precuneus is the key node that interacts directly with all other nodes in the DMN. ${ }^{9,11}$ Deactivation of the precuneus is necessary for focused goal-directed task performance. ${ }^{29}$ The correlation between decreased precuneus activity and milder symptoms observed in this study suggests that reduced DMN connectivity may facilitate deactivation of the precuneus for focused goal-directed task performance in patients with mild iNPH.

In the present study, DMN connectivity was shown to correlate with the severity of cognitive decline. Among the neuropsychological assessment measures used in this study, FAB was the only one to show a significant correlation with DMN connectivity. The FAB score is a measure of attention and executive function. ${ }^{13}$ These results com- 

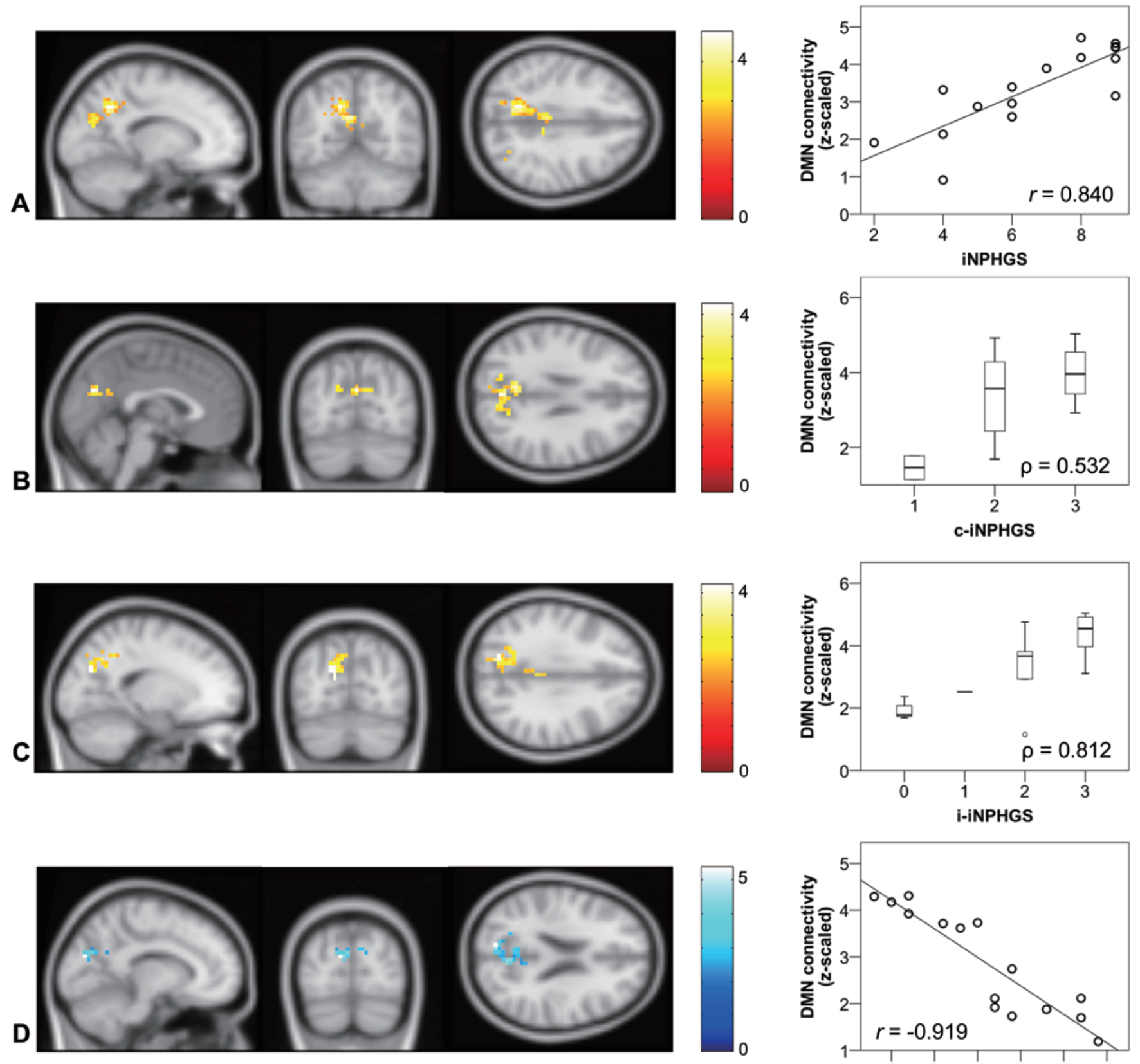

FIG. 5. DMN regions and correlation between DMN connectivity and the severity of clinical symptoms in patients with iNPH. All clusters are thresholded at a whole-brain topological FWE probability of $p<0.05$. A: Cluster of significantly increased connectivity with increased iNPHGS score $(r=0.840)$. B: Cluster of significantly increased connectivity with increased c-iNPHGS score $(\rho=$ 0.532). C: Cluster of significantly increased connectivity with increased i-iNPHGS score $(\rho=0.812)$. D: Cluster of significantly decreased connectivity with increased FAB score $(r=-0.919)$. The significant clusters are overlaid on an MNI152 3.5-mm template in the radiological convention (right-left). Note that the boxplots and scatter plots show the raw data before correction for age.

plement previous findings that the cognitive dysfunction of patients with iNPH is characterized by prominent attention deficit. ${ }^{19,22}$

In the clinical context, DMN connectivity, especially the strength of the connectivity within the precuneus, may be a reliable indicator of a patient's severity of symptoms. Because rs-fMRI studies are easily performed in routine clinical settings, connectivity studies are a potentially useful complement to bedside behavioral assessments for evaluating the severity of iNPH.

Further studies will increase the applicability of our study findings. First, a larger sample size would increase the generalizability of our findings. Second, direct comparison of DMN between patients with iNPH and patients 
with AD or another type of geriatric dementia would yield additional information for differentiating iNPH from these disorders. Third, studies on the interactions between DMN and other large-scale brain networks would improve our understanding of the alterations in functional connectivity in patients with iNPH.

\section{Conclusions}

Our results provide the first evidence for alteration of the DMN in patients with iNPH. We show that DMN connectivity is reduced in patients with iNPH and correlates with the severity of their clinical symptoms. This reduction may reflect a compensatory neural process in patients because of their impairments. Clinically, this study does not clarify the significance of using rs-fMRI to differentiate iNPH from other types of geriatric dementia. However, it is reasonable to suggest that functional connectivity analysis using rs-fMRI may be used preoperatively to provide objective indicators for the severity of symptoms and perhaps help the surgeon in patient selection.

\section{Acknowledgment}

We thank Dr. Mitsuyoshi Takahara for his helpful comments regarding the statistical analysis of this work.

\section{References}

1. Beckmann CF, DeLuca M, Devlin JT, Smith SM: Investigations into resting-state connectivity using independent component analysis. Philos Trans R Soc Lond B Biol Sci 360:1001-1013, 2005

2. Beckmann CF, Smith SM: Probabilistic independent component analysis for functional magnetic resonance imaging. IEEE Trans Med Imaging 23:137-152, 2004

3. Beckmann CF, Smith SM: Tensorial extensions of independent component analysis for multisubject FMRI analysis. Neuroimage 25:294-311, 2005

4. Bonnelle V, Leech R, Kinnunen KM, Ham TE, Beckmann $\mathrm{CF}$, De Boissezon X, et al: Default mode network connectivity predicts sustained attention deficits after traumatic brain injury. J Neurosci 31:13442-13451, 2011

5. Broyd SJ, Demanuele C, Debener S, Helps SK, James CJ, Sonuga-Barke EJ: Default-mode brain dysfunction in mental disorders: a systematic review. Neurosci Biobehav Rev 33:279-296, 2009

6. Damoiseaux JS, Beckmann CF, Arigita EJ, Barkhof F, Scheltens P, Stam CJ, et al: Reduced resting-state brain activity in the "default network" in normal aging. Cereb Cortex 18:1856-1864, 2008

7. Damoiseaux JS, Rombouts SA, Barkhof F, Scheltens P, Stam CJ, Smith SM, et al: Consistent resting-state networks across healthy subjects. Proc Natl Acad Sci U S A 103:1384813853,2006

8. Filippini N, MacIntosh BJ, Hough MG, Goodwin GM, Frisoni GB, Smith SM, et al: Distinct patterns of brain activity in young carriers of the APOE-epsilon4 allele. Proc Natl Acad Sci U S A 106:7209-7214, 2009

9. Fransson P, Marrelec G: The precuneus/posterior cingulate cortex plays a pivotal role in the default mode network: Evidence from a partial correlation network analysis. Neuroimage 42:1178-1184, 2008

10. Greicius MD, Srivastava G, Reiss AL, Menon V: Defaultmode network activity distinguishes Alzheimer's disease from healthy aging: evidence from functional MRI. Proc Natl Acad Sci U S A 101:4637-4642, 2004
11. Hagmann P, Cammoun L, Gigandet X, Meuli R, Honey CJ, Wedeen VJ, et al: Mapping the structural core of human cerebral cortex. PLoS Biol 6:e159, 2008

12. Hawellek DJ, Hipp JF, Lewis CM, Corbetta M, Engel AK: Increased functional connectivity indicates the severity of cognitive impairment in multiple sclerosis. Proc Natl Acad Sci U S A 108:19066-19071, 2011

13. Iavarone A, Ronga B, Pellegrino L, Loré E, Vitaliano S, Galeone F, et al: The Frontal Assessment Battery (FAB): normative data from an Italian sample and performances of patients with Alzheimer's disease and frontotemporal dementia. Funct Neurol 19:191-195, 2004

14. Ishikawa M, Hashimoto M, Kuwana N, Mori E, Miyake H, Wachi A, et al: Guidelines for management of idiopathic normal pressure hydrocephalus. Neurol Med Chir (Tokyo) 48 Suppl:S1-S23, 2008

15. Kay BP, DiFrancesco MW, Privitera MD, Gotman J, Holland SK, Szaflarski JP: Reduced default mode network connectivity in treatment-resistant idiopathic generalized epilepsy. Epilepsia 54:461-470, 2013

16. Kubo Y, Kazui H, Yoshida T, Kito Y, Kimura N, Tokunaga $\mathrm{H}$, et al: Validation of grading scale for evaluating symptoms of idiopathic normal-pressure hydrocephalus. Dement Geriatr Cogn Disord 25:37-45, 2008

17. Lang S, Duncan N, Northoff G: Resting-state functional magnetic resonance imaging: review of neurosurgical applications. Neurosurgery 74:453-465, 2014

18. Mori E, Ishikawa M, Kato T, Kazui H, Miyake H, Miyajima M, et al: Guidelines for management of idiopathic normal pressure hydrocephalus: second edition. Neurol Med Chir (Tokyo) 52:775-809, 2012

19. Ogino A, Kazui H, Miyoshi N, Hashimoto M, Ohkawa S, Tokunaga $\mathrm{H}$, et al: Cognitive impairment in patients with idiopathic normal pressure hydrocephalus. Dement Geriatr Cogn Disord 21:113-119, 2006

20. Raichle ME: Neuroscience. The brain's dark energy. Science 314:1249-1250, 2006

21. Rorden C, Bonilha L, Fridriksson J, Bender B, Karnath HO: Age-specific CT and MRI templates for spatial normalization. Neuroimage 61:957-965, 2012

22. Saito M, Nishio Y, Kanno S, Uchiyama M, Hayashi A, Takagi M, et al: Cognitive profile of idiopathic normal pressure hydrocephalus. Dement Geriatr Cogn Dis Extra 1:202-211, 2011

23. Sánchez-Cubillo I, Periáñez JA, Adrover-Roig D, RodríguezSánchez JM, Ríos-Lago M, Tirapu J, et al: Construct validity of the Trail Making Test: role of task-switching, working memory, inhibition/interference control, and visuomotor abilities. J Int Neuropsychol Soc 15:438-450, 2009

24. Seeley WW, Menon V, Schatzberg AF, Keller J, Glover GH, Kenna H, et al: Dissociable intrinsic connectivity networks for salience processing and executive control. J Neurosci 27:2349-2356, 2007

25. Sharp DJ, Beckmann CF, Greenwood R, Kinnunen KM, Bonnelle V, De Boissezon X, et al: Default mode network functional and structural connectivity after traumatic brain injury. Brain 134:2233-2247, 2011

26. Smith SM, Fox PT, Miller KL, Glahn DC, Fox PM, Mackay $\mathrm{CE}$, et al: Correspondence of the brain's functional architecture during activation and rest. Proc Natl Acad Sci U S A 106:13040-13045, 2009

27. Tian L, Jiang T, Wang Y, Zang Y, He Y, Liang M, et al: Altered resting-state functional connectivity patterns of anterior cingulate cortex in adolescents with attention deficit hyperactivity disorder. Neurosci Lett 400:39-43, 2006

28. Vanhaudenhuyse A, Noirhomme Q, Tshibanda LJ, Bruno MA, Boveroux P, Schnakers C, et al: Default network connectivity reflects the level of consciousness in non-communicative brain-damaged patients. Brain 133:161-171, 2010 
29. Weissman DH, Roberts KC, Visscher KM, Woldorff MG: The neural bases of momentary lapses in attention. Nat Neurosci 9:971-978, 2006

30. Whitfield-Gabrieli S, Ford JM: Default mode network activity and connectivity in psychopathology. Annu Rev Clin Psychol 8:49-76, 2012

\section{Disclosure}

This research was supported by the Grants-in-Aid for Young Scientists (grant no. 24791500), the Brain Mapping by Integrated Neurotechnologies for Disease Studies (Brain/MINDS), and the Strategic Research Program for Brain Science (SRPBS) from the Japanese Ministry of Education, Culture, Sports, Science and Technology, Japan.

\section{Author Contributions}

Conception and design: Kishima, Khoo. Acquisition of data: Kishima, Khoo, Tani, Kazui, Watanabe. Analysis and interpretation of data: Kishima, Khoo, Tani. Drafting the article: Khoo.
Critically revising the article: Kishima, Khoo, Tani, Oshino, Maruo, Hosomi, Yanagisawa, Kazui, Watanabe, Aso, Kawaguchi, Yamashita, Saitoh, Yoshimine. Reviewed submitted version of manuscript: Kishima, Khoo, Oshino, Maruo, Hosomi, Yanagisawa, Kazui, Watanabe, Shimokawa, Aso, Kawaguchi, Yamashita, Saitoh, Yoshimine. Approved the final version of the manuscript on behalf of all authors: Kishima. Statistical analysis: Khoo, Tani, Shimokawa, Kawaguchi, Yamashita. Administrative/technical/ material support: Kishima, Hosomi, Yanagisawa, Watanabe, Aso, Kawaguchi, Yamashita. Study supervision: Kishima, Yoshimine.

\section{Supplemental Information \\ Previous Presentation}

Parts of this work were presented as proceedings at the 15th annual meeting of the Japanese Society of Normal Pressure Hydrocephalus, Osaka, Japan, February 1, 2014.

\section{Correspondence}

Haruhiko Kishima, Department of Neurosurgery, Osaka University Graduate School of Medicine, 2-2, Yamada-oka, Suita, Osaka 565-0871, Japan. email: hkishima@nsurg.med.osaka-u.ac.jp. 